\title{
El paisaje en el proyecto turístico obrero de las Ciudades de Vacaciones de Educación y Descanso
}

\section{The landscape in the worker tourism project of the Holiday cities from the Trade Union Work Education and Rest}

\author{
$\underline{\text { Ricardo Carcelén González }}^{(*)}$
}

\section{RESUMEN}

La Obra Sindical Educación y Descanso promovió en los años 50 un proyecto turístico para la clase obrera de nuestro país con la construcción de tres ciudades de vacaciones en Tarragona, Marbella y Perlora, que mostraron actitudes encaminadas a la preservación y puesta en valor del paisaje natural, desde el propio trazado de los conjuntos hasta el diseño de sus arquitecturas. En este trabajo se analizan dichas actitudes desde un punto de vista tanto cualitativo como cuantitativo, arrojando datos objetivos que reflejan la relación del referido proyecto turístico obrero con el paisaje. Con la llegada de los años 60 y de los grandes planes de ordenación turística de promoción privada, la Obra Sindical se vio obligada a introducir algunas variaciones sobre su modelo vacacional -en su proyecto para Punta Umbría, Huelva- que, como se concluye en este trabajo, conducirá a una manifiesta transgresión de su relación original con el paisaje.

Palabras clave: Obra Sindical Educación y Descanso; Ciudad de vacaciones; Paisaje; Turismo; Clase obrera.

\section{ABSTRACT}

In the 1950s, the Trade Union Work Education and Rest promoted a tourist project for the working class of our country with the construction of three holiday cities in Tarragona, Marbella and Perlora, which showed attitudes aimed at the preservation and enhancement of the natural landscape, from the layout of the cities to the design of their architectures. In this text, these attitudes are analyzed from both a qualitative and quantitative point of view, bringing objective data that reflects the relationship between landscape and over time of the aforementioned worker tourism project. With the arrival of the 196os and the large tourist development plans of private promotion, Education and Rest was forced to introduce some variations on its holiday model-in its project for Punta Umbría, Huelva- which, as can be concluded in this work, it will lead to a manifest transgression of his original relationship with the landscape.

Keywords: Trade Union Work Education and Rest; Holiday cities; Landscape; Tourism; Worker class.

(*) Arquitecto. Profesor Contratado Doctor. Universidad Politécnica de Cartagena (España).

Persona de contacto/Corresponding author: ricardo.carcelen@upct.es (R. Carcelén González).

ORCID: http://orcid.org/oooo-0o02-8266-8797 (R. Carcelén González).

Cómo citar este artículo/Citation: Ricardo Carcelén González (2021). El paisaje en el proyecto turístico obrero de las Ciudades de Vacaciones de Educación y Descanso. Informes de la Construcción, 73(564): e415. https://doi.org/10.3989/ic.81316

Copyright: (C) 2021 CSIC. Este es un artículo de acceso abierto distribuido bajo los términos de la licencia de uso y distribución Creative Commons Reconocimiento 4.0 Internacional (CC BY 4.0). 
1. EL DERECHO AL REPOSO DEL OBRERO Y EL DESEO DE LA ORILLA

El fenómeno turístico en Europa experimentó una de sus grandes transformaciones cuando la clase proletaria alcanzó el derecho a un descanso anual remunerado. La clase trabajadora tenía por primera vez opción a experimentar, aunque tan solo fuese por tiempo limitado, los privilegios de aquella clase ociosa que en 1899 nos presentaba el sociólogo estadounidense Veblen (1) para referirse a esa parte de la población reputada y respetada por su ostensible abstención del trabajo. Por fin, la clase obrera se había rebelado contra aquel amor incondicional que profesaba hacia el trabajo, y que Lafargue denunciaba abiertamente en su alegato en favor del derecho a la pereza (2), o Russell algunas décadas después en aquel ensayo en el que elogiaba a la ociosidad (3), y donde alentaba a los obreros a considerar el trabajo tan sólo como el medio ineludible para ganarse el sustento, a la vez que les exigía reclamar su derecho al reposo, a disfrutar de su tiempo libre.

Con la consecución del derecho al descanso anual remunerado, la clase obrera europea logra su ansiada ociosidad, momento en el que se materializa la gran transformación a la que antes nos referíamos, cuando el hasta entonces turismo elitista y aristocrático, sólo al alcance de minorías, se convierte en un turismo de masas, un derecho al reposo al alcance de las clases sociales con menos recursos, con el que recuperar fuerzas antes de volver nuevamente al desempeño del trabajo (4). Quizás, por ello, algunos autores se empeñan en desterrar el término de turismo de masas en favor del término turismo popular, para referirse de manera más explícita al fenómeno turístico específico de este momento histórico en el que la clase obrera comienza a poner en práctica su derecho a las vacaciones pagadas (5).

En España, la clase obrera tuvo que recorrer un dilatado trayecto hasta hacerse de manera definitiva con ese derecho a unas vacaciones anuales retribuidas (6). Esta demanda histórica se abordó por primera vez en la Constitución Republicana del año 1931 -en su artículo 46-, y se consolidó algunos años después con la promulgación del Fuero del Trabajo de 1938 -en su declaración II, artículos 5 y 6y en la posterior Ley del Contrato del Trabajo de 1944 -en su capítulo IV, artículo 35-, lo que llevó al régimen franquista a idear un proyecto turístico que se ocupase de la organización del ocio y las vacaciones de los trabajadores del país. Algunos autores insisten en un interés profundo del Régimen por transformar el turismo en un fenómeno de índole social, alejándose así de los proyectos y propuestas de carácter elitista y minoritario (7), y que desencadenaría una honda transformación en la sociedad española de la que los medios de prensa de la época se hacían eco (8).

A partir de aquellas experiencias similares llevadas a cabo previamente por otros regímenes totalitarios en Italia, Alemania o Portugal, entre otros, el organismo estatal competente en estos términos, la Obra Sindical Educación y Descanso, instauró en España una compleja red de albergues y residencias de veraneo con localizaciones diversas a lo largo y ancho de la geografía del país, resultando significativo el número de instalaciones de este tipo emplazadas en el ámbito litoral. Este hecho no es casual, sino que encuentra su raison d'être en el "deseo de la orilla" o el "anhelo de la ribera" al que se refería el historiador Corbin (9), cuando nos relataba el paulatino interés del ser humano por el mar, a pesar de partir de un sentimiento primigenio de absoluta repulsión hacia éste. Autores como Boyer (10), o posteriormente Larrinaga (11) para el caso concreto de España donde el fenómeno se dio con casi un siglo de retraso respecto a Europa, continuarán el legado del historiador francés abordando este campo de investigación en las épocas más recientes.

El rotundo éxito que entre las masas trabajadoras cosechó la labor desempeñada por Educación y Descanso durante sus dos primeras décadas de existencia, propició el escenario idóneo para la implementación de un novedoso modelo para la organización, así como también el control, del descanso y el ocio estival de los productores españoles: las ciudades de vacaciones de Educación y Descanso. Se trataba de un proyecto turístico mucho más ambicioso que el de las residencias marinas o las estaciones balnearias ensayadas en los litorales italiano y alemán respectivamente. El propio José Solís Ruiz, Delegado Nacional de Sindicatos entre 1951 y 1957, proclamaba en la prensa contemporánea su interés por una serie de "poblaciones residenciales para el descanso del trabajador", en las que el paisaje natural, encarnado en su propio imaginario por "risueñas playas y un pinar sano y frondoso" (12), resultaba fundamental.

\section{LAS CIUDADES DE VACACIONES DE EDUCA- CIÓN Y DESCANSO}

Promovidas a mediados de la década de los años cincuenta, la Obra Sindical Educación y Descanso construyó en España hasta tres ciudades para las vacaciones de los trabajadores, ejecutadas ad hoc como instalaciones autosuficientes estratégicamente localizadas en privilegiadas parcelas en primera línea de mar, de tal manera que toda la longitud del litoral de nuestro país quedaba dividido en porciones equivalentes con el fin de minimizar los desplazamientos de los trabajadores por la geografía española (13) (figura 1), y destinadas no solo a proporcionar descanso durante el asueto estival de sus residentes, sino también como medida de adoctrinamiento político que de manera velada se ponía en práctica en el distendido ambiente que allí se generaba, un escenario que propiciaba el discurso franquista a favor de la familia y de los valores del cristianismo, en total sintonía con la propaganda del Régimen (14). Al fin y al cabo, esta modalidad vacacional garantizaba el aislamiento del trabajador y de sus familias durante sus vacaciones, casi como si nos encontrásemos ante un "secuestro de los viajeros" (15).

No cabe duda de que, gracias a ello, los mandos de estas instalaciones podían cumplir su función de adoctrinamiento político sin mayores dificultades, "tan solo conversando con los obreros de tú a tú" (16). Pero no se puede obviar, y así se trata de poner de manifiesto en este trabajo, la búsqueda intencionada de emplazamientos aislados en el medio natural para alejarse en la medida que fuera posible de los poco saludables núcleos de población donde los trabajadores residían todo el año, a lo que algunos autores se refieren como un "entendimiento de las vacaciones como sinónimo de descanso en un entorno natural privilegiado con límites legibles" (17), adelantando de esta manera una de las características que de manera unánime satisfacían las ciudades de vacaciones de Educación y Descanso. 

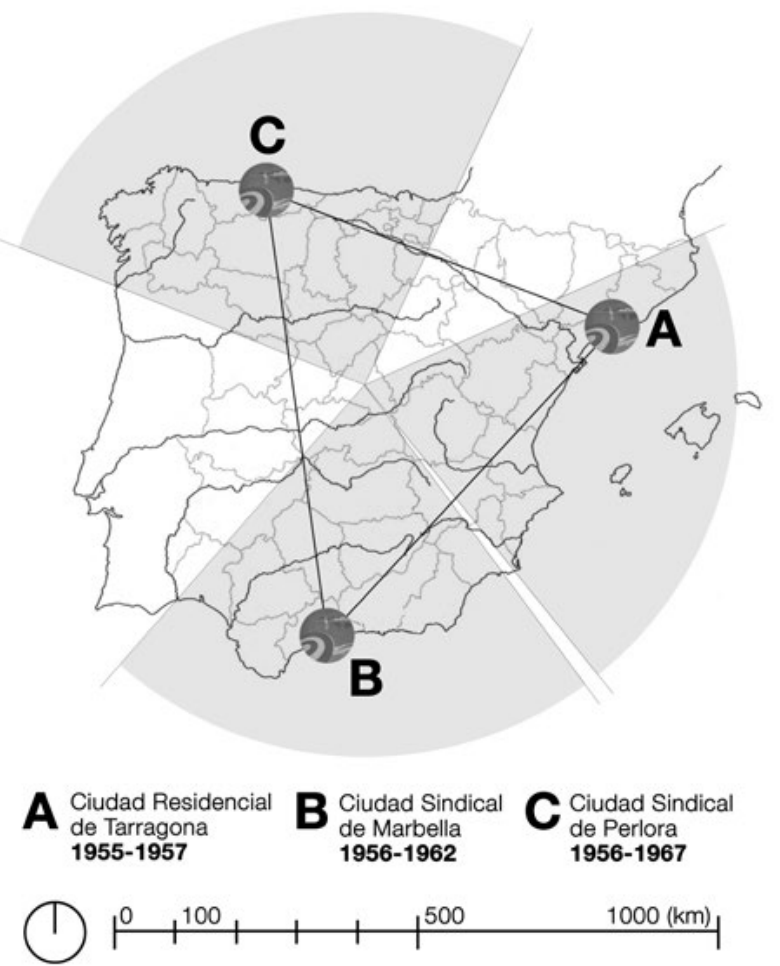

Figura 1. Situación estratégica de los conjuntos. Fuente: Autor, a partir de Carcelén 2019a (18) y Carcelén 2019b (19).

La de la Playa Larga de Tarragona, ideada en 1955 por los arquitectos Josep María Monravà López y Antoni Pujol Sevil (figura 2), supuso la creación de un nuevo modelo vacacional no sólo en nuestro país, sino en toda Europa. Los terrenos, de propiedad pública, fueron directamente ofrecidos a la Obra Sindical por el entonces Gobernador Civil y Jefe Provincial en Tarragona, D. José González-Sama García, uno de los principales interesados en el éxito del proyecto (20).

A pesar de la libertad de planteamientos inicial que se otorgó a los técnicos para la redacción del proyecto urbano y arquitectónico, éstos debieron contemplar algunas instrucciones extraoficiales (desconocidas al ser verbales) del referido González-Sama (21). Además de las evidentes ventajas cualitativas respecto del modelo inicial de residencias, entre las que habría que destacar la posibilidad de los trabajadores de disfrutar de las vacaciones en familia, razones cuantitativas se encontraban detrás de este ambicioso proyecto turístico de Educación y Descanso, que trataba con estos conjuntos de dar solución a la cada vez mayor demanda por parte de los trabajadores que aspiraban a pasar en ellos sus vacaciones estivales. Los técnicos catalanes asumieron el reto de crear una nueva ciudad de la nada, con la dificultad añadida de tener que idear "un novedoso programa de ocio y descanso previo a la inminente expansión turística que sufrieron nuestras costas en la década de los años sesenta” (22).

De ese esfuerzo sacaría rédito Educación y Descanso cuando en febrero de 1956 publica la convocatoria del concurso para el diseño de la nueva ciudad de vacaciones en Marbella, en cuyo segundo punto se enumeraba el régimen de necesidades mínimas a satisfacer en el conjunto, redactado claramente desde el amplio conocimiento que la experiencia de la construcción del conjunto tarraconense había conferido a la organización sindical, y en su punto tercero se rechazaban explícitamente los proyectos o ideas que pudieran considerarse "antiestéticos o de contraste con la zona y las características de la región” (23). De la materialización del conjunto de Marbella se encargarían finalmente los arquitectos Manuel Aymerich Amadios y Ángel Cadarso del Pueyo (figura 3), en colaboración con los estudiantes de arquitectura Ignacio Gárate y Alfonso Crespo, y con los arquitectos José M. Arangüena y Calixto del Barrio, quienes se hicieron con la victoria en el concurso de ideas al que nos referíamos.

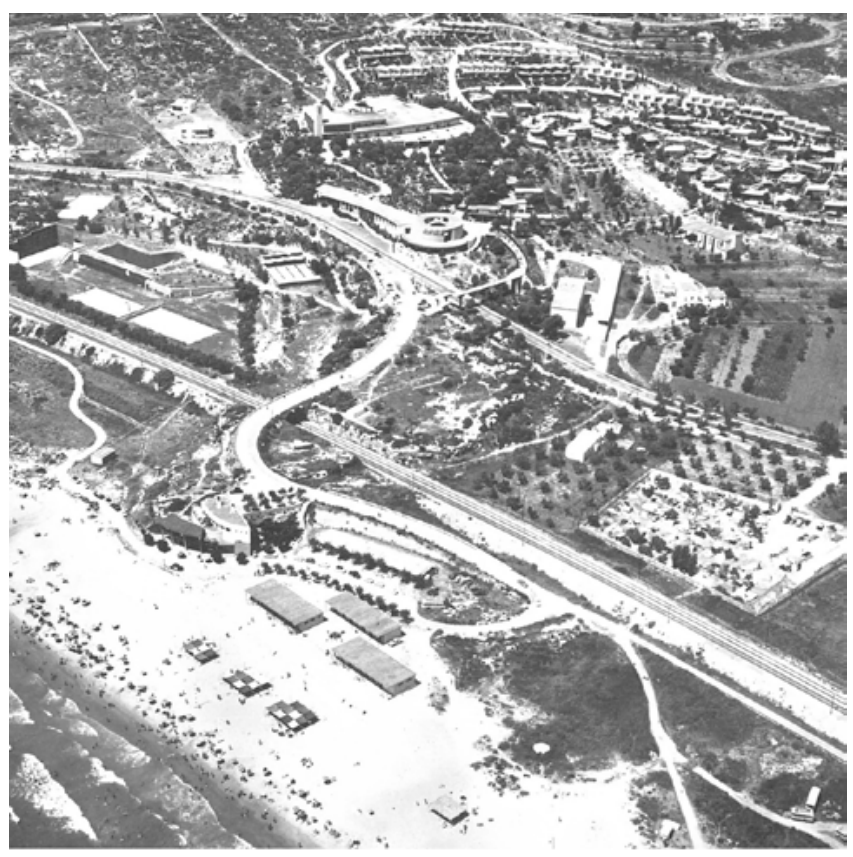

Figura 2. Ciudad de vacaciones en Tarragona. Fuente: F/33-04445o3 (Archivo General de la Administración, AGA).

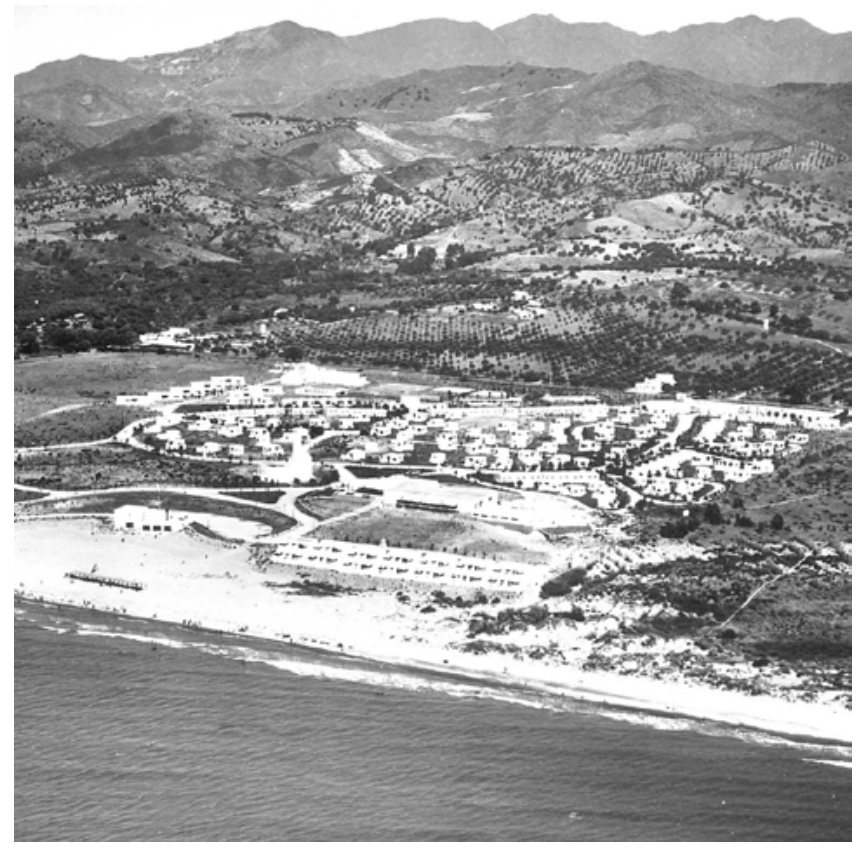

Figura 3. Ciudad de vacaciones en Marbella. Fuente: F/33-04564o7 (AGA).

Ya en diciembre de 1955, la prensa nacional informaba en sus páginas de la firma de las escrituras de los terrenos que la Diputación Provincial de Málaga habría adquirido, con el fin de cederlos a la Delegación Nacional de Sindicatos para la ejecución de esta segunda ciudad (24). 
Simultáneamente a la construcción de la anterior, se sucederían los trabajos de ejecución de una tercera ciudad residencial de vacaciones en Perlora, Oviedo, bajo la supervisión de los hermanos Federico y Francisco Somolinos Cuesta (figura 4), reproduciendo el programa que tanto éxito había cosechado en Tarragona. Con la finalización de los conjuntos de Marbella y Perlora a principio de la década de los 60, quedaba consolidada una sutil avanzadilla de la posterior depredación que acusaría el litoral español en la década posterior. En relación con los terrenos, en este caso de propiedad privada, la Delegación Provincial de Sindicatos y la Diputación Provincial tuvieron que llevar a cabo las pertinentes gestiones para la adquisición de los $350.000 \mathrm{~m}^{2}$ requeridos para la ejecución de este tercer conjunto, entonces "distribuidos en un total de 129 parcelas, de 182 propietarios y colonos diferentes” (25).

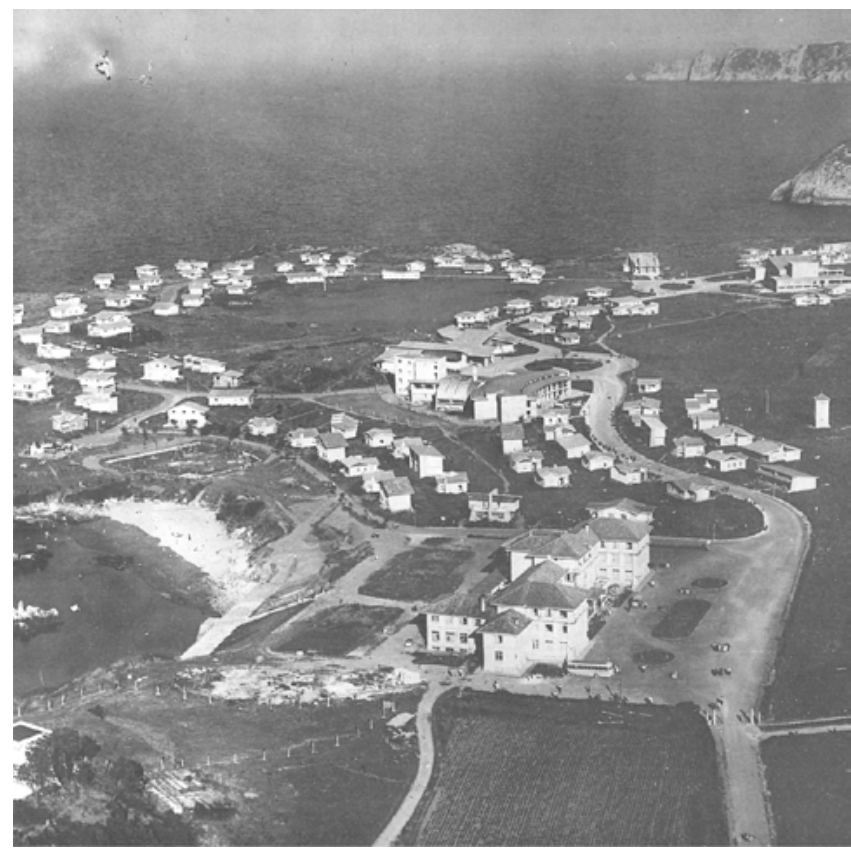

Figura 4. Ciudad de vacaciones en Perlora. Fuente: F/33-04592-11 (AGA).

Además del programa arquitectónico, integrado fundamentalmente por piezas para la residencia temporal de los trabajadores y sus familias, y por equipamientos colectivos (comedores, iglesia, tiendas, recepción, ...) para garantizar la autosuficiencia de los conjuntos, las tres ciudades de vacaciones de Educación y Descanso compartían como seña de identidad la elección de localizaciones privilegiadas junto al mar para su construcción (26). Esa condición especial de los emplazamientos fue posible fundamentalmente por el estado casi virginal que se encontraron los arquitectos responsables de los diferentes conjuntos cuando iniciaron sus trabajos en la década de los años cincuenta (27). Frente a esos paisajes inmaculados, los conjuntos de Educación y Descanso tomarían una serie de actitudes comunes que se desarrollan a continuación (28).

\section{ACTITUDES FRENTE AL PAISAJE}

Partiendo de lo irrepetible de la situación paisajística de partida de estos conjuntos vacacionales (figura 5), la respuesta que Educación y Descanso dio con su proyecto turístico de las ciudades de vacaciones mostró una especial sensibilidad por el paisaje y el entorno natural (29), basándose en el aprovechamiento máximo de las características naturales del terre- no así como de sus singularidades topográficas; en el respeto por la vegetación y el arbolado preexistentes; así como en el empleo de arquitecturas muy dialogantes con el paisaje en que habrían de insertarse (30). Recordemos que una de las premisas de las ciudades de vacaciones consistía en provocar el desplazamiento de los trabajadores de las ciudades insalubres a localizaciones enclavadas en unos territorios naturales en los que paisaje, clima, realidad litoral, ... constituían el soporte territorial cuya integridad, continuidad e identidad natural debía ser salvaguardado para no entrar en contradicciones.
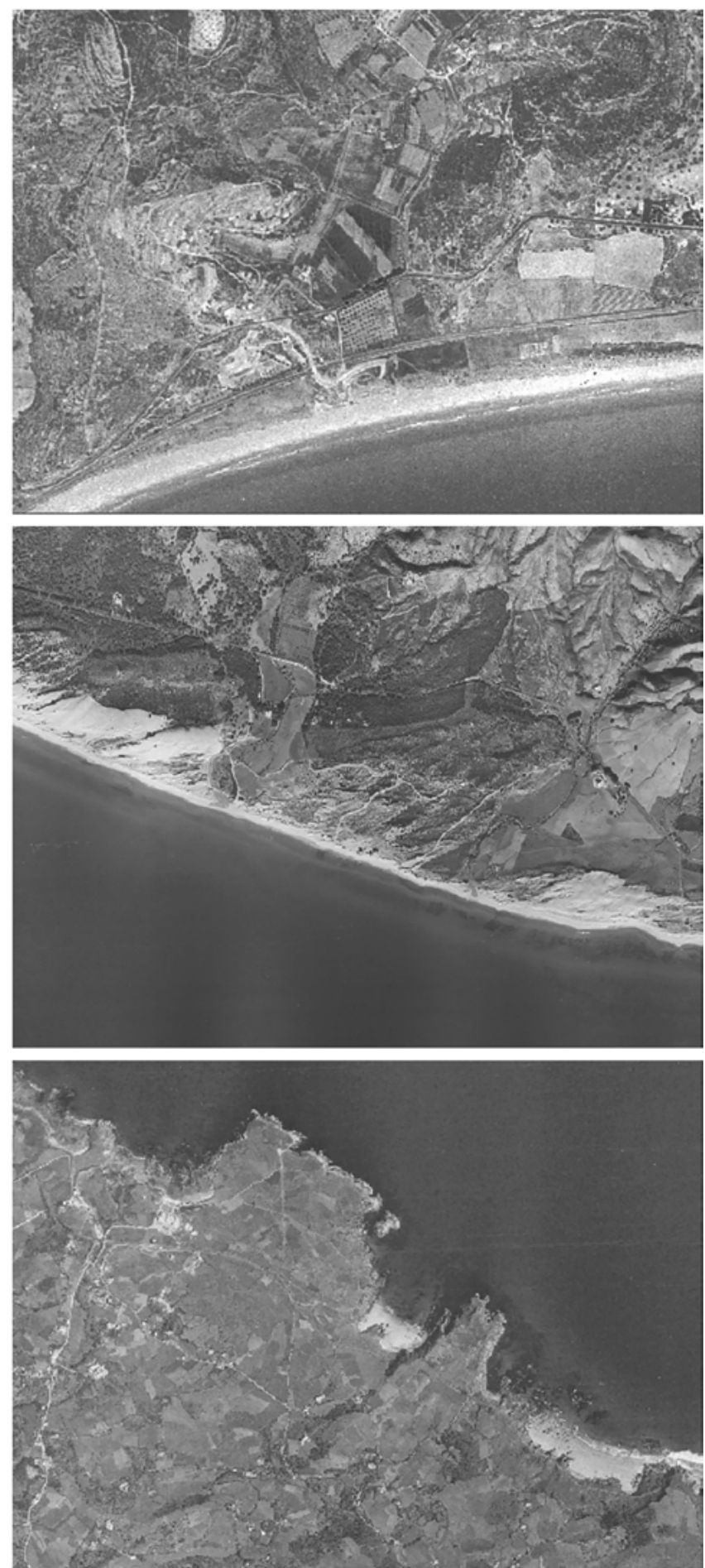

Figura 5. Implantación en el entorno natural. Fuente: Fotogramas del vuelo Americano Serie-B, 1956-1957, Instituto Geográfico Nacional (IGN). 
Algunas corrientes de estudio sobre el paisaje construido (31), hablan de un proceso de ajuste entre la obra y el entorno en el que se inserta, que pasa por "analizar los conceptos de localización (dónde se ubica), configuración espacial (cómo la disponemos), articulación (cómo expresar su relación con el entorno), y fronteras y vínculos (hasta dónde llega)". En ese sentido, y para determinar el ajuste entre las ciudades de vacaciones de Educación y Descanso y el emplazamiento natural donde se emplazaron, en este trabajo se propone el análisis cuantitativo y/o cualitativo de los siguientes conceptos relacionados con los anteriores: la capacidad de integración en el paisaje y la adaptación al lugar (localización); los trazados generales y los skylines de los conjuntos (configuración espacial); el estudio de vacíos, el análisis de uso de los espacios exteriores así como intermedios, y las características específicas de las arquitecturas de los conjuntos (articulación); y, finalmente, los límites específicos (fronteras y vínculos).

\subsection{Integración en el paisaje y adaptación al lugar}

Los tres conjuntos de Educación y Descanso se caracterizan por la atenta lectura del lugar que los arquitectos responsables en cada caso llevaron a cabo, con especial atención a la orografía de los emplazamientos y a la presencia de elementos preexistentes de origen natural a preservar. Sin embargo, los emplazamientos originales presentaban condiciones tan dispares que iban desde el fuerte desnivel constante en Tarragona (desnivel máximo de 46 metros), a la suave orografía en Marbella que se transformaba en abrupta al tomar contacto con la playa (20 metros de desnivel máximo), y finalmente, a la gran llanura en Perlora, con algunos promontorios puntuales en su perímetro (19 metros de desnivel máximo). Las coincidencias entre las tres ciudades de vacaciones se encuentran, entonces, en la respuesta dada con la implantación de los proyectos en el terreno, sin interponer en ningún caso ni la urbanización ni la arquitectura a la topografía natural (figura 6).

La actitud en realidad sería a la inversa, influyendo radicalmente las condiciones específicas del emplazamiento sobre muchas de las decisiones tomadas en cada uno de los proyectos, afectando incluso al propio diseño de algunas de las edificaciones cuyas secciones se adaptaban al perfil de terreno. Así lo evidenciaban, por ejemplo, los arquitectos Monravà y Pujol en la memoria de proyecto, al señalar que "el estudio general del proyecto se ha verificado partiendo de la base de adaptar al terreno los edificios constituyentes de esta ciudad residencial, ya que el mismo reúne unas condiciones naturales difícilmente superables, para la finalidad para la que fue adquirido". En Tarragona, el diseño aprovechaba el fuerte desnivel del terreno para garantizar las visuales hacia el mar desde todas las piezas que integraban la zona residencial del conjunto.

En el caso de Marbella, el fuerte desnivel existente en la duna en contacto con la playa propiciaba esa misma situación, favoreciendo el diseño escalonado de las edificaciones residenciales en ese ámbito. O la localización del comedor colectivo en la única elevación puntual del terreno, en busca de visibilidad y visuales, aprovechando así las condiciones naturales de la parcela. En Perlora, la presencia de dos promontorios en el emplazamiento repercutió de manera directa en la orientación conferida a algunas de las piezas del conjunto, que buscaban sortear estos obstáculos visuales hacia el mar. No se trataba por tanto de proyectos prototípicos, sino de propuestas específicas para unos lugares concretos.
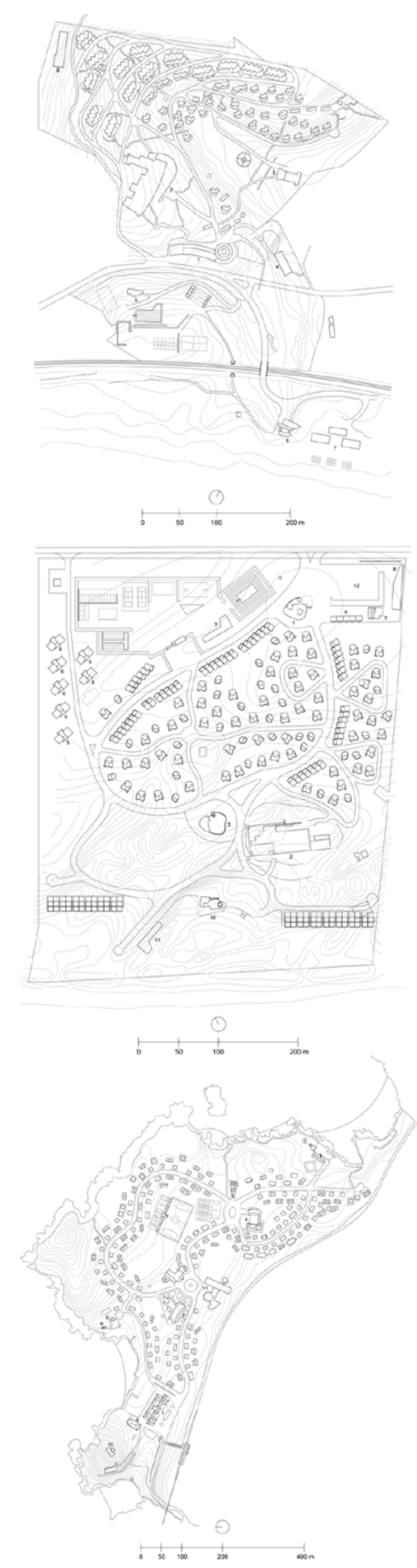

Figura 6. Planos de ordenación general. Fuente: Autor. 
Los conjuntos de Educación y Descanso interpretaron el terreno de implantación como un tapiz natural frente al que dieron una respuesta común de mínima urbanización, potenciando en la medida de lo posible los valores paisajísticos y naturales propios del lugar. En casos más extremos como el del conjunto de Marbella, la preservación de la vegetación y el arbolado preexistentes en la parcela condicionarían la propia implantación de las edificaciones, así como el trazado de los viales del conjunto tal y como indican en la memoria de proyecto los propios arquitectos.

\subsection{Ciudades de trazado orgánico}

Los trazados en los conjuntos de Educación y Descanso se ajustan en todos los casos a esquemas de tipo orgánico (figura 7), según apuntaban los arquitectos responsables de los proyectos, por puras cuestiones de adaptación a la topografía original, de ruptura con cualquier posible monotonía en el conjunto, y de la cotizada búsqueda de vistas hacia el mar. Ese carácter orgánico estará reforzado, en los tres casos, por la predominancia de unos trazados viarios, rodados y peatonales, con esos mismos criterios, donde se daba además una jerarquización de estos, identificándose siempre un viario principal que generaba la estructura organizativa del conjunto, y un segundo orden de viarios secundarios, que conferirían a la ciudad la permeabilidad y la libertad de recorridos deseable para este tipo de instalaciones. Aquellos puntos en los que confluían los viarios principales se convertían en puntos estratégicos al ser ocupados en todos los casos por los equipamientos colectivos principales de este modelo, actuando como nodos de actividad: el edificio de recepción y dirección, el comedor (o comedores) y, finalmente, la iglesia.

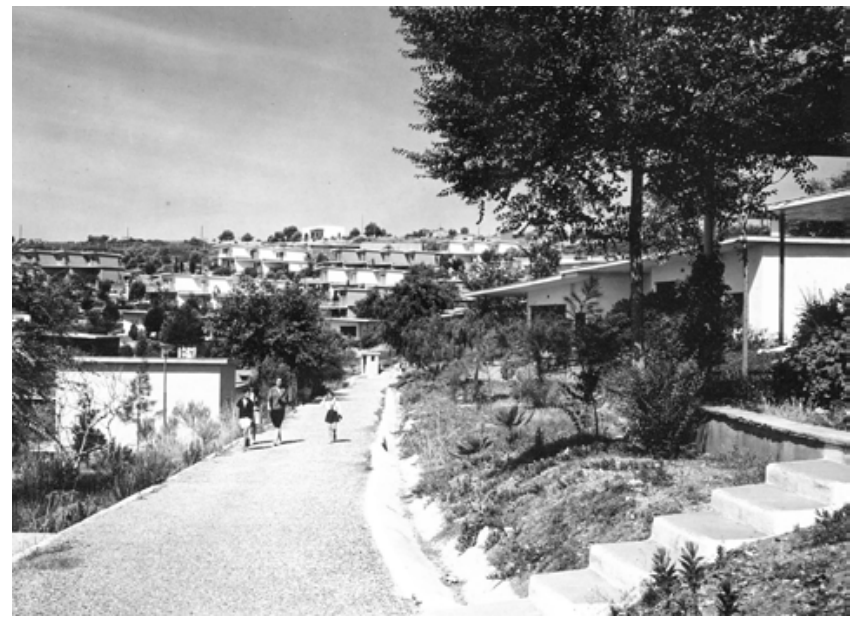

Figura 7. Trazados orgánicos en Tarragona. Fuente: F/33-04445-O3 (AGA).

En Tarragona, los trazados viarios requieren de la curva para proporcionar a los senderos el recorrido apropiado que garantice una pendiente cómoda para el tránsito de los residentes en su descenso diario hasta la playa, así como el correspondiente camino de retorno al apartamento al finalizar la jornada. En Marbella, los trazados viarios se desarrollan de forma orgánica para proteger, según los arquitectos responsables, la vegetación preexistente en la parcela, a la vez que cuando se recorren se van obteniendo visuales hacia el mar a través de los vacíos existentes entre las diferentes edificaciones que integran el conjunto. En Perlora, también los viarios se ajustan a trazados orgánicos.
A pesar sin embargo de la similitud de planteamientos, la repercusión que el viario tiene sobre el total de la superficie ocupada por los tres conjuntos será dispar, condicionado en gran medida por las condiciones naturales de la orografía original del terreno. Esto es lo que provoca que en Tarragona, que acusaba el mayor desnivel de los tres casos, el viario representara más de la quinta parte de la superficie total de parcela, con un 22,1\% de ocupación de la misma, quedando los valores de Marbella (13,7\%) y Perlora (11,3\%) muy por debajo de esa cifra.

\subsection{Skyline de baja altura}

Las ciudades de vacaciones ejecutadas en Tarragona, Marbella y Perlora se caracterizan por su rebajado skyline, referido a la reducida altura de las edificaciones que los integran y, por tanto, a la reducida altura global del conjunto. En todos ellos se opta por resolver el programa arquitectónico en una o dos plantas, dando lugar a unas instalaciones caracterizadas por su planicidad. Esta condición es la que propicia que los equipamientos colectivos sean las únicas piezas que destacan en los conjuntos, ya sea porque en estos se recurra a mayor altura que las dos plantas, o porque como en el caso de las iglesias algunos de sus elementos verticales -el campanarioactúan como hitos visuales del paisaje.

\subsection{El predominio del vacío}

El vacío representa una de las principales características de las ciudades de vacaciones de Educación y Descanso, propiciado sin lugar a duda por lo innecesario de especular con los terrenos ante el tipo de uso y el carácter público y oficial de cada una de estas promociones. Esto nos lleva a unos valores superiores a los $200 \mathrm{~m}^{2}$ de suelo libre -de terreno natural y paisaje- por cada residente usuario de estos conjuntos, con la salvedad de Tarragona donde el valor se ve penalizado por la fuerte repercusión de la ocupación del viario (Tarragona: 77,86 $\mathrm{m}^{2} /$ residente; Marbella: 212,56 $\mathrm{m}^{2} /$ residente; Perlora: $204,40^{2} /$ residente). En esa misma línea del predominio del vacío, este modelo vacacional se caracterizará por la baja densidad de los conjuntos, con valores medios alrededor de las 10 viviendas por hectárea (Tarragona: 13,4viv/ha; Marbella: 7,3viv/ha; Perlora: 7,88viv/ha).

En términos de ocupación, encontramos que las ciudades de vacaciones de Educación y Descanso presentan un valor medio de suelo libre de uso público de en torno al 75\% (Tarragona: 67,1\%; Marbella: $77 \%$; Perlora: $80,5 \%$ ), lo que se traduce en que al menos las tres cuartas partes de la superficie de estos conjuntos se destinaba a este tipo de uso del suelo. Si lo que se analiza es el suelo libre de uso privado, es decir, aquel suelo que aún estando libre de edificación se encuentra asociado a una vivienda a modo de patio privativo, el valor medio obtenido es casi residual (sólo en las instalaciones de Marbella la figura del patio adquiere cierta importancia, con un $2,8 \%$ de ocupación para este tipo de uso del suelo). El reducido valor para este último parámetro pone de manifiesto la idea de unos conjuntos permeables y de libre recorrido, donde la ausencia de vallados y delimitaciones de parcela refuerzan el valor de lo colectivo.

\subsection{La apropiación del exterior}

El hecho de que este tipo de instalaciones se utilizasen, al menos al principio de su puesta en funcionamiento, exclusi- 
vamente durante los meses de verano, provocaba que los residentes hiciesen un elevado uso de los espacios exteriores, tanto los de sus propias unidades residenciales temporales como los de los equipamientos de uso colectivo. Patios, porches, terrazas, etcétera, en definitiva, lo que entendemos como espacios intermedios, serán recurrentes en las piezas arquitectónicas de las ciudades de vacaciones de Educación y Descanso, tratando de alcanzar una deseada prolongación de los espacios interiores en el exterior. Para ello, los arquitectos responsables de estas arquitecturas recurrirán al empleo de grandes paños acristalados en la mayoría de las edificaciones, condicionados tan solo por las limitaciones técnicas de la época.

Si se analiza en términos cuantitativos, la cifra de superficies útiles de las unidades residenciales destinadas a este tipo de espacios intermedios respecto de la superficie construida total de alojamientos de los conjuntos, se obtienen valores ciertamente elevados que confirman la idea anterior (Tarragona: $8.066,97 \mathrm{~m}^{2}$-superficie total construida de unidades residenciales- frente a $6.654,42 \mathrm{~m}^{2}$-superficie total de espacios exteriores-; Marbella: $9.685,8 \mathrm{om}^{2}$ frente a $8.100,81 \mathrm{~m}^{2}$; Perlora: $13.248,58 \mathrm{~m}^{2}$ frente a $3.974,70 \mathrm{~m}^{2}$ ).

De igual manera, los equipamientos de uso colectivo también se apropiarán del espacio exterior. En algunos casos extremos, como en las iglesias, se aprovecharán las favorables condiciones climatológicas del verano litoral para plantear edificaciones que de manera alternativa a su uso interior ofrecían la posibilidad de presenciar las liturgias desde el espacio exterior, bien mediante el empleo de grandes explanadas al aire libre frente a las iglesias, bien por la delimitación de su propio espacio exterior como ocurre en Marbella -en reformas posteriores, este espacio al aire libre ha sido transformado en espacio interior cubierto, desvirtuando la idea original-, ampliando así el aforo de estas piezas.

\subsection{La terraza como alternativa al patio}

El empleo de terrazas en planta baja o planta superior de forma generalizada -salvo en contadas excepciones, como aquellas unidades residenciales en Marbella que por ocupar posiciones interiores dentro del conjunto y ante la imposibilidad de lograr visuales de interés, optan por el uso de patios- para que los usuarios pudieran disfrutar de vistas lejanas hacia el mar condicionará la imagen formal de estos conjuntos (figura 8). También en las piezas destinadas a equipamiento colectivo se recurrirá al uso de terrazas, fundamentalmente en Tarragona y Marbella donde las condiciones climatológicas resultaban más favorables, y donde las amplias terrazas exteriores orientadas hacia el mar en los comedores comunitarios serían el reclamo de los trabajadores. Esto resultaba especialmente efectivo en aquellos bares-restaurantes que se situaban en las zonas marítimas de los conjuntos, sobre la propia arena de las playas.

\subsection{Arquitectura moderna adaptada al lugar}

Las arquitecturas que integraban los conjuntos vacacionales de Educación y Descanso eran fundamentalmente equipamientos de uso colectivo (por lo general edificio de recepción, comedor/es, edificio de relación y administración, centro médico o enfermería, tiendas o galería comercial, edificio destinado a garaje-taller, y, finalmente, iglesia) y unidades de alojamiento temporal en múltiples tipologías entre apartamentos, bungalows aislados, viviendas pareados o adosadas, bloques escalonados, etcétera (en Tarragona 180 unidades
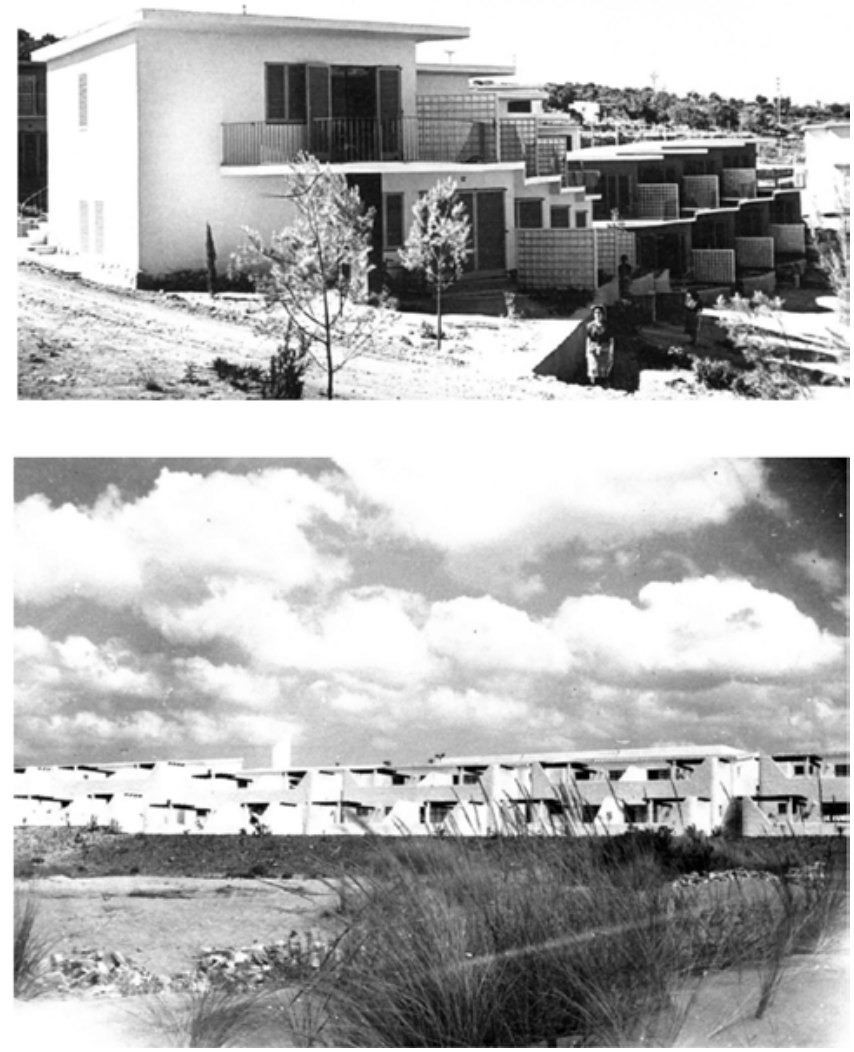

Figura 8. Uso de terrazas (Tarragona, arriba; Marbella, abajo). Fuente: F/33-04587-04 y F/33-04592-08 (AGA).

para una capacidad máxima en un único turno de 1152 trabajadores; en Marbella 199 unidades para una capacidad de 829 trabajadores por turno; y en Perlora 274 unidades para una capacidad total de 1.370 residentes).

Las diferentes piezas arquitectónicas construidas en las ciudades de vacaciones de Educación y Descanso pueden enmarcarse en aquel estilo arquitectónico que algunos autores denominan como moderno adaptado o moderno contextualizado, un lenguaje en el límite que separa tradición y modernidad. Así, los arquitectos responsables de cada uno de los diseños en Tarragona, Marbella y Perlora encontraron en este estilo la manera idónea para integrar sus modernas arquitecturas en el territorio, en el paisaje donde se implantaban, ayudándose para ello del uso de materiales de construcción autóctonos -superficies de chinarro encalado en Marbella o la piedra y la madera en Perlora-.

A este respecto, las ciudades de vacaciones de Educación y Descanso supusieron un punto de inflexión en la producción arquitectónica de la organización sindical destinada al ocio $\mathrm{y}$ al reposo de los trabajadores, que hasta entonces se identificaba más con los valores de la arquitectura de corte tradicional -basta con revisar la propia residencia de veraneo Jacobo Campuzano en Perlora, que sirvió de germen para la posterior ejecución de la ciudad de vacaciones que aquí se está tratando-.

\subsection{La playa como límite}

Las ciudades de vacaciones de Educación y Descanso coincidirán en su posición cercana a la playa, al mar, que en el peor 
de los casos no superará los 600 metros de distancia para los puntos más desfavorables de cada conjunto, ni los 50 metros para los más próximos.

Los tres conjuntos tendrán en común la forma en la que acometían el contacto con el límite configurado por la orilla litoral. En todos ellos, el límite se vuelve impreciso. La transición entre los conjuntos y la arena de la playa se desvanece, acercándose al planteamiento de las colonias de vacaciones italianas, prescindiendo de paseos marítimos. En el proyecto turístico de las ciudades de vacaciones de Educación y Descanso no había cabida para este elemento, de carácter más urbano, que tanta aceptación tendría en el contexto del turismo de sol y playa de décadas después. Sólo cuando la costa era más abrupta, como en el caso de los acantilados de Perlora, se hacía necesaria la intervención arquitectónica, aunque solo para hacer posible el recorrido de descenso de los trabajadores hacia la playa.

Tampoco el resto de los límites de estos conjuntos vacacionales serían más precisos, con la inexistencia de un viario perimetral de ronda que definiese los límites de actuación, las tres ciudades de vacaciones parecen desparramarse más allá de sus límites, fundiéndose con el paisaje natural. Sólo los trazados de infraestructuras terrestres, cuya vinculación con los conjuntos resultaba imprescindible para garantizar la subsistencia de unos conjuntos que se ejecutaban como autosuficientes, parecían definir verdaderos límites en cada una de las actuaciones. La línea del ferrocarril y la carretera $\mathrm{N}-340$ en Tarragona determinarían la propia separación del conjunto en diferentes áreas funcionales. De nuevo la N-340, ahora a su paso por Marbella, definía el límite opuesto a la playa. En Perlora, el trazado del tren asumiría ese papel.

\section{EL CAMBIO DE IDEALES EN PUNTA UMBRÍA: LA TRANSGRESIÓN DEL PAISAJE}

A finales de la década de los años 6o, el que fuera secretario General de Educación y Descanso, Federico Olivencia Amor, declaraba en prensa en favor de la actividad desarrollada hasta la fecha por la Obra Sindical, haciendo hincapié entre otros aspectos en el parque inmobiliario de residencias y ciudades de vacaciones ejecutadas, y anunciando la futura construcción de hasta dos nuevos conjuntos siguiendo la estela de Tarragona, Marbella y Perlora, en las localidades de Guardamar del Segura -Alicante- y Punta Umbría -Huelva-.

Lo cierto es que esas declaraciones llegan en un contexto en el que el paisaje del litoral español ya se encontraba sometido a un proceso de agresiva colonización, y que anticipaba la sobreexplotación de la franja costera de nuestro país. Ese consumo del territorio y del paisaje como mera mercancía sería lo que caracterizaría la etapa desarrollista del franquismo, impulsada en parte desde el propio Estado por la ley de Centros y Zonas de Interés Turístico Nacional promovida por el Ministerio de Información y Turismo con Manuel Fraga a los mandos, y por otra parte por la promoción privada, ambos posicionados en favor no sólo de incentivar la llegada del turismo internacional de masas a nuestro país, sino también de ofrecer una modalidad de veraneo al alcance de la clase trabajadora del país como alternativa a la que hasta entonces ofertaba casi en exclusividad Educación y Descanso. En este nuevo contexto al que nos referimos, la Obra Sindical abordará el proyecto de las dos nuevas ciudades de vacaciones para trabajadores, de las cuales se conocen detalles del proyecto desarrollado en 1968 para las playas de Punta Umbría (figura 9), proyectado por el arquitecto onubense Francisco de la Corte López, en calidad de asesor de la Obra Sindical del Hogar y Arquitectura en Huelva.

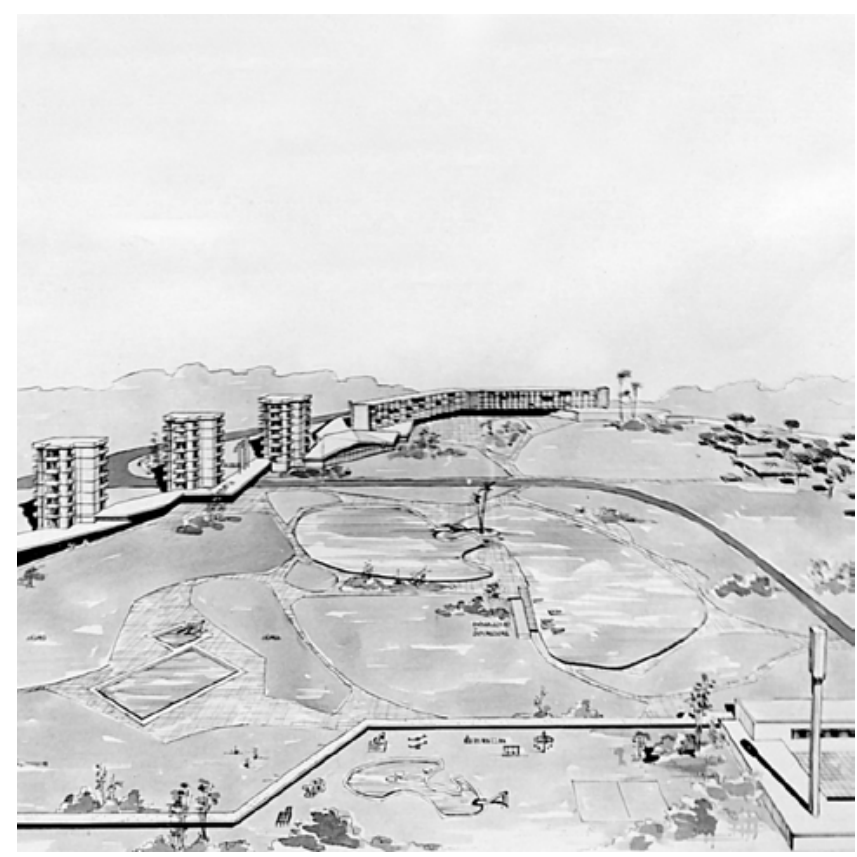

Figura 9. Ciudad de vacaciones en Punta Umbría, Huelva. Fuente: F/33-04609-33 (AGA).

Para la ejecución de este nuevo conjunto, Educación y Descanso opta por primera vez por un emplazamiento no situado en primera línea de playa, en terreno público cedido mediante concesión administrativa acordada en Pleno del Ayuntamiento de Punta Umbría de febrero de 1969 (32), con distancias mínimas hasta el mar que no descienden en ningún caso de 600 metros, una medida que ni en el peor de los casos se alcanzaba para los puntos más desfavorables en Tarragona, Marbella o Perlora (aquí, el punto más cercano respecto del mar se encontraba a 605 metros medidos en la perpendicular, y el más alejado a poco más de un kilómetro de distancia),. La playa deja así de ser uno de los límites fundamentales en el conjunto, renunciando por tanto a uno de los mayores atractivos para los usuarios de este tipo instalaciones.

Tampoco en Punta Umbría parece ya ser tan importante ni la integración paisajística ni la adaptación al terreno, obviándose de partida en la materialización del proyecto de ordenación general del conjunto tanto la topografía como las condiciones propias de la parcela, o la propia preservación de la vegetación y arbolado habitual, a juzgar por la existencia de informes de la Sección Forestal de Huelva de la Subdirección de Montes y Política Forestal, de la Dirección General de Montes Caza y Pesca Fluvial, ante la solicitud formal del arquitecto para la tala de algunos de los pinos preexistentes en las inmediaciones del emplazamiento, con el fin de trazar un acceso secundario al mismo.

Con relación a los trazados en este último conjunto, sí se mantiene la tendencia a la mínima urbanización, con valores para la ocupación del viario por debajo del 12\% (cercano a los valores de Marbella, 13,7\%, y Perlora, 11,3\%), y al empleo de desarrollos orgánicos, quizás aquí más por imitación de sus predecesoras que por la adaptación a la topografía o por la 
búsqueda de visuales hacia el mar, imposibles por la posición interior de la parcela respecto de la orilla.

También se aplican criterios similares cuando se analiza el espacio libre del conjunto onubense, donde el vacío sigue siendo el gran protagonista, con valores de $144,57 \mathrm{~m}^{2}$ de suelo libre por cada residente (superando a Tarragona con 77,86 $\mathrm{m}^{2} /$ res, la cifra queda lejana de los $212,56 \mathrm{~m}^{2} /$ res y 204,40 $\mathrm{m}^{2} /$ res de Marbella y Perlora respectivamente). En términos de ocupación de suelo, tanto los espacios libres de uso público como los de uso privado siguen la senda de Tarragona, Marbella y Perlora, con valores muy elevados para el primer caso $(75,6 \%$, en la línea del valor medio del $75 \%$ de las tres antecesoras) y casi nulos $(0,1 \%)$ para el segundo (en Tarragona y Perlora recordemos que el valor era nulo, y apenas del 2,8\% de la ocupación en Marbella). Sin embargo, si atendemos a parámetros de densidad, en la ciudad de vacaciones de Punta Umbría casi se duplica el valor medio que se señalaba para sus predecesoras (10 viviendas por hectárea), con valores en torno a 17,5 viviendas por hectárea de actuación.

El skyline rebajado, seña de identidad del proyecto turístico para trabajadores de Educación y Descanso, se desvirtúa por primera vez ante el empleo de edificación en altura de la edificación residencial, que provoca tanto la ruptura de la horizontalidad del nuevo paisaje como la pérdida de representatividad de los equipamientos públicos, haciendo que se resientan los habituales hitos arquitectónicos ya sean las torres campanario o los escultóricos depósitos de agua en los conjuntos de la década anterior.

La apropiación del espacio exterior en Punta Umbría no resulta tan evidente como en Tarragona, Marbella o Perlora, y cuando hace acto de presencia, se cede su uso para aparcamiento de los automóviles utilitarios de los trabajadores. Tan sólo en las edificaciones residenciales en altura resulta significativa la presencia de terrazas, que sin embargo no ofrecían disposiciones óptimas en lo que a visuales hacia el mar se refiere, a la vez que veían muy reducida sus superficies en comparación con los casos de estudio pioneros.

Desafortunadamente, la ciudad de vacaciones en Punta Umbría no llegaría a materializarse finalmente, al menos como conjunto residencial, aunque sí como residencia, al reorientarse el objeto del proyecto a la construcción de un hotel-residencia de Educación y Descanso, todavía en funcionamiento en la actualidad como Residencia de Tiempo Libre. A pesar de ello, la volumetría de conjunto elaborada por el arquitecto Francisco de la Corte nos presenta una arquitectura que, aunque quizás en sintonía con los valores arquitectónicos del momento, adolece de esa imagen de arquitectura enraizada en la cultura y el paisaje propios del lugar.

\section{CONCLUSIONES}

Como conclusión, trataremos de esbozar cuales pudieron ser las razones que llevaron a Educación y Descanso a mostrar actitudes más transgresoras con el paisaje en su proyecto turístico obrero más tardío.

Con la llegada de la década de los años 6o, se produce como vimos el distanciamiento no sólo físico, sino también visual, de las ciudades de vacaciones de Educación y Descanso respecto del mar y la playa. Ya señalamos como para el conjunto de Punta Umbría la Obra Sindical optó por un emplazamiento interior, más alejado de la orilla litoral de lo que venía siendo habitual para este modelo vacacional. La justificación a dicho distanciamiento podría encontrarse en que en ese nuevo contexto de ocupación masiva de nuestras playas, serían las grandes operaciones turísticas de orden privado las que se disputarían esas primeras posiciones en el paisaje (en definitiva, las que podrán permitirse la adquisición de esos terrenos), privilegiadas desde el punto de vista de las visuales hacia el mar y el contacto con la playa, que encontraban en estos valores la oportunidad de ofertar destinos turísticos de mayor atractivo no solo para la clase trabajadora del país, sino también para el turista internacional. Esa evidente pérdida de competitividad de los conjuntos de Educación y Descanso respecto de las promociones privadas, podría ser la causa de algunas de las transgresiones de este proyecto turístico obrero con relación al paisaje, como proponer la construcción de un gran lago artificial para paliar el alejamiento respecto del mar, a pesar de que ello demostrase una menor sensibilidad hacia las condiciones naturales preexistentes del lugar de implantación.

Así, la necesidad de generar conjuntos competitivos llevaría a la Obra Sindical a incrementar la densidad de sus ciudades de vacaciones con la incorporación de edificación residencial en altura, en el caso de Punta Umbría, materializada con el bloque en altura de una residencia-hotel así como seis torres de apartamentos para el alojamiento de los trabajadores, que definitivamente acabarán con ese skyline al que se ajustaban los conjuntos en Tarragona, Marbella y Perlora, y que contribuía a conservar la horizontalidad del paisaje donde se insertaban.

El aumento del tamaño de las unidades residenciales, así como la incorporación de cocinas y amplios salones de estar en Punta Umbría, derivó también en una menor intensidad en la apropiación de los espacios exteriores, que de alguna manera representaba la estrategia fundamental mediante la cual la arquitectura de estos conjuntos establecía un diálogo directo con el lugar y con el paisaje. El incremento de los espacios interiores, por tanto, provocaría la reducción de estos espacios intermedios -fundamentalmente terrazas- que se habían erigido como fundamentales en los conjuntos de la década de los años 50.

Finalmente, y también en esa línea de ofertar destinos de vacaciones competitivos, Educación y Descanso se vio irremediablemente abocada a introducir cambios drásticos en el programa de sus ciudades de vacaciones, un aspecto que había permanecido invariable en los tres conjuntos de Tarragona, Marbella y Perlora. En Punta Umbría se introdujeron nuevos usos -también se prescindió de otros, como la iglesia- como amplios salones para la celebración de congresos o auditorios, que supusieron una auténtica novedad para este modelo turístico obrero, como consecuencia probablemente de las influencias ejercidas por los grandes planes de ordenación turística que al mismo tiempo se estaban desarrollando en nuestro país. De nuevo, esa necesidad de ser competitivos llevó a la obra Sindical a considerar incluso en Punta Umbría la construcción de un puerto deportivo que, junto con los usos anteriores, no resultan en consonancia con las demandas de la clase obrera de nuestro país, en definitiva, destinatario potencial de este tipo de conjuntos.

Con todo esto, se ha tratado de poner de manifiesto la paradoja de cómo en la búsqueda de destinos de vacaciones más competitivos, y de haberse llegado a materializar el conjunto de Punta Umbría, el proyecto turístico obrero de Educación y Descanso 
objeto de este trabajo habría experimentado una evidente pérdida de relación con el paisaje respecto a sus antecesoras, lo que en sí mismo representó en origen uno de los grandes logros de este modelo vacacional. Es a esto precisamente a lo que Aguiló (33) se refiere cuando nos habla del "turismo como aproximación empobrecedora”, y de cómo los atractivos previos deben permanecer intactos pues, de lo contrario, con su disminución y eliminación desaparece su idoneidad para el turismo.

Concluido este trabajo, resulta ciertamente difícil de precisar las razones que llevaron a Educación y Decanso al abandono del proyecto turístico de sus ciudades de vacaciones (materializado en el desistimiento de los planes para Guardamar del Segura y Punta Umbría), si bien se exponen a continuación algunas de las causas que podrían haber motivado un cambio en la estrategia del régimen franquista.

Por un lado, no es extraño que ante la imposibilidad de amortizar la fuerte inversión económica que suponía la ejecución de estas instalaciones por su destino a fines sociales, sumada a la dificultad de competir con la alta densidad asumida por los modelos especulativos que se desarrollarían en el seno del turismo de masas durante la etapa desarrollista del país -en la que la baja densidad claramente no resultaba competitiva-, la Obra Sindical ensayase otras fórmulas alternativas a priori más económicas, basadas en la explotación en régimen de alquiler de instalaciones privadas, como los también malogrados Complejos Sindicales de Turismo Social. O, en el extremo totalmente opuesto, a promocionar un turismo internacional de masas que ya en la década de los sesenta comenzaba a posicionarse como uno de los principales motores de desarrollo económico del país.

Por otro lado, no debe descartarse tampoco que ese abandono del modelo turístico se debiera a un acusado descenso en la demanda de estas instalaciones de veraneo de Educación y Descanso por parte de la clase obrera de nuestro país, como consecuencia de un importante incremento en la oferta derivado de los motivos que se exponían anteriormente.

Probablemente, la solución a la incógnita deba buscarse en una suma de ambas circunstancias (34).

\section{REFERENCIAS}

(1) Veblen, T. (1944). Teoría de la clase ociosa (The Theory of the Leisure Class. An Economic Study of Institutions. Macmillan Company. Primera edición en inglés: 1899). México: Fondo de Cultura Económica.

(2) Lafargue, P. (2004). El derecho a la pereza. Madrid: Editorial Fundamentos.

(3) Russell, B. (2000). Elogio de la ociosidad. Barcelona: Editorial Edhasa.

(4) Pié i Ninot, R. (2013). Las arquitecturas del turismo: las piezas mínimas. En R. Pié i Ninot y C. J. Rosa Jiménez (Eds.), Turismo líquido (pp. 16-39). Barcelona: Instituto Hábitat Turismo Territorio a través de Iniciativa Digital Politècnica UPC, Universitat Politècnica de Catalunya y Universidad de Málaga. Recuperado de https://upcommons.upc.edu/handle/2099.3/36559

(5) Couveia, A. A. (1995, 16-17 de noviembre). Identité edt encadrement international du tourisme social. Trabajo presentado en el Seminario Internacional de Turismo Social, Calvià, Mallorca.

(6) Muñiz Aguilar, D. (2001). La política del Turismo Social. Málaga: Consejería de Turismo y Deporte, Junta de Andalucía.

(7) De Ortueta Hilberath, E. (2007). Tarragona destino turístico. El patrimonio cultural y los nuevos espacios para el ocio. Revista Norba-Arte (XXVII), 263-284.

(8) El veraneo de los productores. La Vanguardia Española, 15/o6/1957: 6.

(9) Corbin, A. (1993). El territorio del vacío. Occidente y la invención de la playa (1750-1840). Barcelona: Editorial Mondadori.

(10) Boyer, M. (2002x). El turismo en Europa, de la Edad Moderna al siglo XX. Historia Contemporánea (25), 13-31. Recuperado de https://www.ehu.eus/ojs/index.php/HC/article/view/5916/5596.

(11) Larrinaga Rodríguez, C. (2002). El turismo en la España del siglo XIX. Historia Contemporánea (25), 157-179. Recuperado de https://www.ehu.eus/ojs/index.php/HC/article/view/5930/5610.

(12) Diario Español en Tarragona, o8/03/1955: 7.

(13) Tomillo Castillo, A. y Sanz Díaz, C. (2012, 14-16 de junio). Las ciudades sindicales en el periodo franquista. Presentado en la International Conference Intervention Approaches for the 2oth Century Architectural Heritage, Madrid.

(14) Tomillo Castillo, A. (2014, 9-10 de mayo). La dialéctica entre progreso y desarrollo en la Arquitectura Moderna Española: el caso de las ciudades sindicales y el tiempo funcional. Trabajo presentado en el I Congreso Nacional de Arquitectura Pioneros de la Arquitectura Moderna Española: vigencia de su pensamiento y obra, Madrid.

(15) Moragues Cortada, D. (2006). Turismo, Cultura y Desarrollo. Madrid: Agencia Española de Cooperación Internacional. Dirección General de Relaciones Culturales y Científicas.

(16) Díaz Bello, (1999, 7-9 de julio). En busca del obrero: la organización del consentimiento en la España Franquista. Trabajo presentado en el II Congreso de Historia Local de Aragón, Huesca.

(17) Loren Méndez, M. (2008). La modernidad Española como relato de las periferias. Laboratorio arquitectónico y visions urbanas en el alejado sur íbero. Apuntes $(21,2), 234-251$. Recuperado de http://www.scielo.org.co/pdf/apun/v21n2/ v21n2a07.pdf.

(18) Carcelén González, R. (2019). Ciudades de vacaciones de Educación y Descanso: cuando la clase obrera española se hizo turista. PASOS. Revista de Turismo y Patrimonio Cultural, 17 (5), 1049-1063. https://doi.org/10.25145/j.pasos.2019.17.073.

(19) Carcelén González, R. (2019). Punta Umbría: the fourth holiday city of "Education and Rest" in Spain. VLC arquitectura. Research Journal, 6 (2), 89-119. https://doi.org/10.4995/vlc.2019.10692.

(20) Diario Español en Tarragona, 22/12/1954: 1.

(21) Guerrero Fernández, J. (2016). Ciudad Residencial de Educación y Descanso de Tarragona: 1954-1959. La organización del ocio obrero en la posguerra Española (Tesina de Máster). Universitat Politècnica de Catalunya. http://hdl. handle.net/2117/89460 
(22) Carcelén González, R. (2017). Cuando la clase obrera se hizo turista. Las ciudades de vacaciones de la Obra Sindical de Educación y Descanso. Estudio de un modelo inacabado 1955-1975 (Tesis Doctoral). Universidad Politécnica de Cartagena. https://doi.org/10.31428/10317/6031

(23) Bases del Concurso para Proyecto de una ciudad residencial en Marbella (Málaga). Archivo Histórico del Colegio Oficial de Arquitectos de Tarragona. Expediente 00600.

(24) La Vanguardia Española, 24/12/1955: 3.

(25) Obra Sindical Educación y Descanso (1956). Ciudad Residencial de Perlora. Oviedo: OSED.

(26) Sanz Díaz, C. (2001). La Ciudad Residencial de Perlora: espacio de ambigüedad territorial, social, ideológica y constructiva. Trabajo presentado en el Congreso Dos Décadas de Cultura Artística en el Franquismo (1936-1956), Granada.

(27) Gavilanes Vélaz de Medrano, J. (2012). El viaje a la Costa del Sol (1959-1969). Proyecto y transformación (Tesis Doctoral). Universidad Politécnica de Madrid.

(28) Carcelén González, R. Op. cit. supra nota 22.

(29) Méndez Baiges, M. (2012). Arquitectura, ciudad y territorio en Málaga (190o-2011). Málaga: Geometría Asociación Cultural.

(30) Nebot Gómez de Salazar, N. (2012). El tour de las marinas. De la invención a la regeneración del paisaje (Tesis Doctoral). Universidad de Málaga.

(31) Aguiló Alonso, M. (1999). El paisaje construido. Una aproximación a la idea de lugar. Madrid: Editorial Castalia.

(32) ABC, Edición Andalucía, 24/10/1971: 51.

(33) Aguiló Alonso, M. Op. cit. supra nota 31, p. 288.

(34) Carcelén González, R. Op. cit. supra nota 22. 